\title{
Improved acoustic signals discrimination using fractional Fourier transform based phase-space representations
}

\section{Zeev Zalevsky ${ }^{\text {a }}$, David Mendlovic ${ }^{\text {a,* }}$, M. Alper Kutay ${ }^{\text {, }}$, Haldun M. Ozaktas ${ }^{\text {c }}$, Jonathan Solomon ${ }^{\text {a }}$}

a Department of Electrical Engineering, Faculty of Engineering, Tel-Aviv University, 69978 Ramat-Aviv, Tel-Aviv, Israel

b TUBITAK-UEKAE Ataturk Bulvari 221, Kavaklidere 06100 Ankara, Turkey

' Department of Electrical Engineering, Bilkent University, TR-06533 Bilkent, Ankara, Turkey

Received 26 September 2000; received in revised form 10 January 2001; accepted 16 January 2001

\begin{abstract}
In this communication we propose performing two-dimensional correlation operation between phase-space representations based on the fractional Fourier transform, instead of correlating the signals themselves. A numerical examples clearly indicates superior discrimination performance. (ㅇ 2001 Published by Elsevier Science B.V.
\end{abstract}

Keywords: Fractional Fourier transform; Phase-spaces; Acoustic signals

\section{Introduction and background}

The fractional Fourier transform (FRT) [1-3] operation has been found useful for various spatial filtering and signal processing applications $[4,5]$. The transform is defined through the transformation kernel ([1]):

$\left\{\mathscr{F}^{a} f\left(u^{\prime}\right)\right\}(u)=\int_{-\infty}^{\infty} B_{a}\left(u, u^{\prime}\right) f\left(u^{\prime}\right) \mathrm{d} u^{\prime}$

where $B_{a}\left(u, u^{\prime}\right)$ is the kernel of the transformation and $a$ is the fractional order.

${ }^{*}$ Corresponding author. Fax: +972-3-642-3508.

E-mail address: mend@eng.tau.ac.il (D. Mendlovic).

$$
\begin{aligned}
B_{a}\left(u, u^{\prime}\right)= & \frac{\exp [-\mathrm{i}(\pi \operatorname{sgn}(\sin \alpha) / 4-(\alpha / 2))]}{|\sin \alpha|^{\frac{1}{2}}} \\
& \times \exp \left[\mathrm{i} \pi \frac{u^{2}+u^{\prime 2}}{\tan \alpha}-2 \mathrm{i} \pi \frac{u u^{\prime}}{\sin \alpha}\right], \\
\alpha=\frac{a \pi}{2} &
\end{aligned}
$$

The relationship of the FRT to optical propagation and optical systems has been discussed in several works [2,3,6-8]. Further references may be found in Ref. [1].

The phase-space representations employed in this communication were first suggested in Ref. [9], where they were referred to as the $(x, p)$ chart and the $(r, p)$ chart, for reasons that will become obvious shortly. Their optical implementation was presented in Ref. [10]. The Cartesian representation of a signal $f(u)$ (the $(x, p)$ chart) is defined by $[9,10]$ :

$T_{f}(u, a)=\left\{\mathscr{F}^{a} f\left(u^{\prime}\right)\right\}(u)$ 
where $\left\{\mathscr{F}^{a} \ldots\right\}$ is the FRT of order $a$. This chart is a two-dimensional (2-D) chart for a one-dimensional (1-D) input signal.

Many new mathematical properties of these representations and their relationship to other phasespace representations are presented in Ref. [11].

The FRT has found many applications in correlation, matched filtering, and pattern recognition [12]. In this transform the amount of shift variance may be controlled by choosing the proper fractional order $a$ for the transformation. When the fractional order is one, the FRT becomes the conventional Fourier transform which is totally shift variant. For fractional order of zero the FRT gives the input function, i.e. it is totally shift invariant. For any other fractional orders in between, the transform has a partial amount of shift variance.

(a)

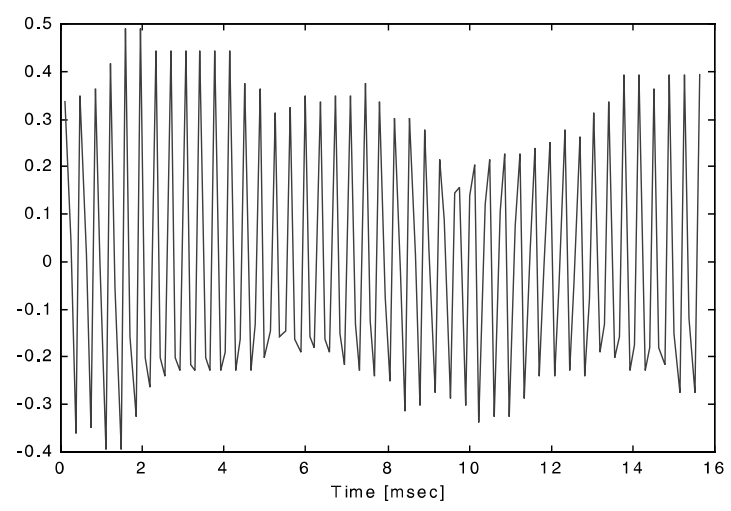

(b)

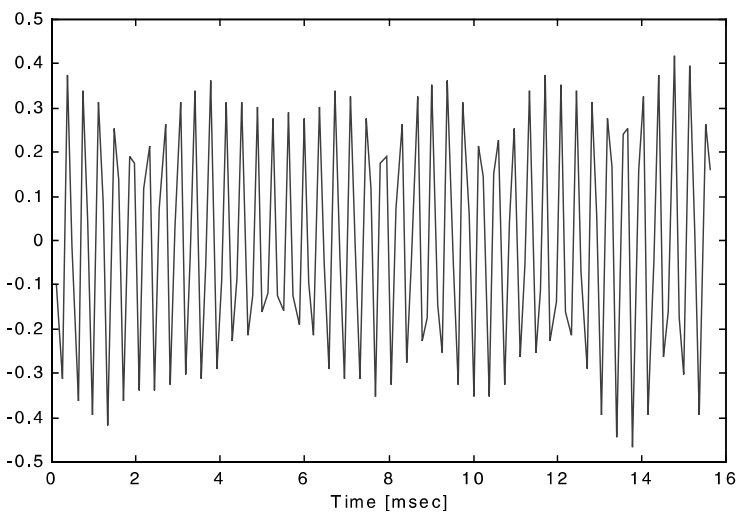

Several researchers have exploited this property for pattern recognition applications (see Ref. [12] for references). Here we will propose a new method based on the phase-space representations introduced above, which is based on correlating these representations instead of the signals themselves.

First, the representation of a reference signal $h(u)$ is computed and stored. Then, we calculate the correlation between the phase-space representations of the incoming signal $f(u)$ with the reference $h(u)$ :

$C(u, a)=\int_{-\infty}^{\infty} \int_{-\infty}^{\infty} T_{f}\left(u^{\prime}, a^{\prime}\right) T_{h}^{*}\left(u^{\prime}-u, a^{\prime}-a\right) \mathrm{d} u^{\prime} \mathrm{d} a^{\prime}$

Numerical evidence indicates that such an algorithm allows greatly superior discrimination re-

(c)

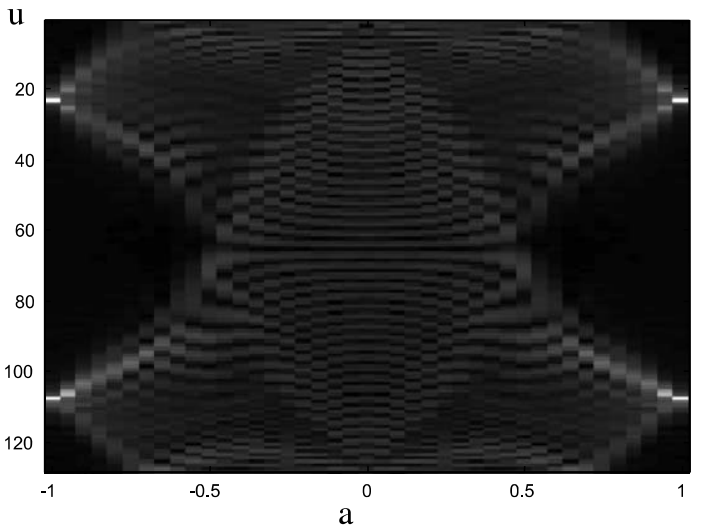

(d)

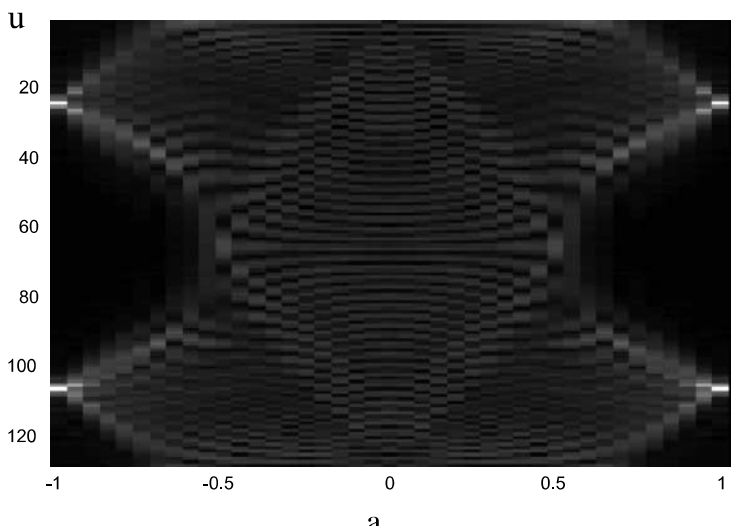

a

Fig. 1. (a) Reference signal $h(u)$, (b) a second, different signal $f(u)$, (c) $T_{h}(u, a)$ for the reference signal $h(u)$ and (d) $T_{f}(u, a)$ for the second signal $f(u)$. [15]. 
sulting from the additional dimension $a$, as we now illustrate with a specific example in Section 2. Some computation considerations as well as the required optical configuration are presented in Section 3. Section 4 concludes the paper.

\section{Computer simulations}

Fig. 1a shows a 128 pixel (15.625 ms) segment from the middle of a chirp acoustic signal sampled at a rate of $8192 \mathrm{~Hz}$. This segment is taken as the reference signal. Fig. 1c shows the Cartesian representation of this reference signal (an $(x, p)$ chart). Fig. 2a presents the correlation given by Eq. (4) when the input signal is the same as the reference. This 2-D correlation expression has two axes - the vertical one that corresponds to the coordinate $u$ and the horizontal one that corresponds to the coordinate $a$. A cross section of this 2-D correlation may be seen in Fig. 2c that displays the cross section at the vertical axis $(a=0)$. For comparison, the correlation between the reference signal and a second similar but distinct acoustic signal (shown in Fig. 1b) is presented in Fig. 2b. Fig. 1d shows its Cartesian representation. The cross section of this correlation shown in Fig. 2d exhibits a much smaller peak. Finally, in Fig. 2e, we show the direct ordinary time-domain correlation of the reference signal with itself and in Fig. 2f, we show the direct ordinary time-domain correlation of the reference signal with the second distinct signal. The peak obtained is much less distinct and highly oscillatory. Overall, it is clear that the discrimination that can be obtained from parts e and $\mathrm{f}$ is poorer than the one obtained from parts $\mathrm{c}$ and $\mathrm{d}$ of Fig. 2. This detection discrimination ability improvement is obtained due to the addition of the another axis: the fractional order $a$.

The underlying reason why the representations employed in this communication lead to superior results may have similar reasons to the benefits obtained by the use of wavelet transforms. In fact, a relationship between the FRT and a certain wavelet family has been pointed out in Ref. [5]. The fractional order axis is equivalent to the scale axis of the wavelet transform since varying the fractional order of the transform scales the chirp kernel which multiplies the signal. As in the wavelet transform, displaying a one dimensional signal using two axes of scale and shift allows to represent the signal properly despite its fast localized frequency variations (as occurs for instance in oscillating or transient signals). A representation that could not be achieved using a conventional spectral display (a Fourier transform). The adaptation between the localization of the input signal and the transformation kernel is the basis for its improved discrimination ability of transient signals (such as acoustic signals).

In order to further demonstrate the ability of the discussed approach, we present additional simulations obtained with various input signals. The presented examples are just a chosen set out of a larger variety of simulations we performed. In Fig. $3 a$ and $b$, one may see two samples of laugh signals. Signal 3a is chosen to be the reference. Fig. 3c and d display the Cartesian representation of the signals in Fig. $3 \mathrm{a}$ and $\mathrm{b}$ respectively. The obtained two dimensional auto and cross correlations are presented in Fig. 3e and $\mathrm{f}$ respectively. Their corresponding cross sections may be seen in Fig. $3 g$ and $h$. As one may notice the auto correlation peak is sharp and the cross correlation is very low. The obtained auto and cross correlations when an ordinary time domain approach is used may be seen in Fig. $3 \mathrm{i}$ and j. Here, the auto correlation is not sharp and the cross correlation is not as low as in Fig. $3 g$ and $h$.

As previously noted, the usage of the suggested phase space allowed to improve the recognition performances of 1-D acoustic signals due to the fact that the phase space is 2-D and thus it has an increased discrimination ability. In addition, the phase space representation, that is FRT based, actually realized a sophisticated wavelet-like spectral decomposition [5]. Wavelet transform capabilities in dealing with transient signals was previously proven [13].

Note that since the investigated phase-space representations are based upon the FRT, they are shift variant. Applying various techniques such as a decomposition of the input signal to circular, Mellin or logarithmic harmonics [14] prior to computing the phase space may allow incorporating invariant pattern recognition capabilities. 
(a)

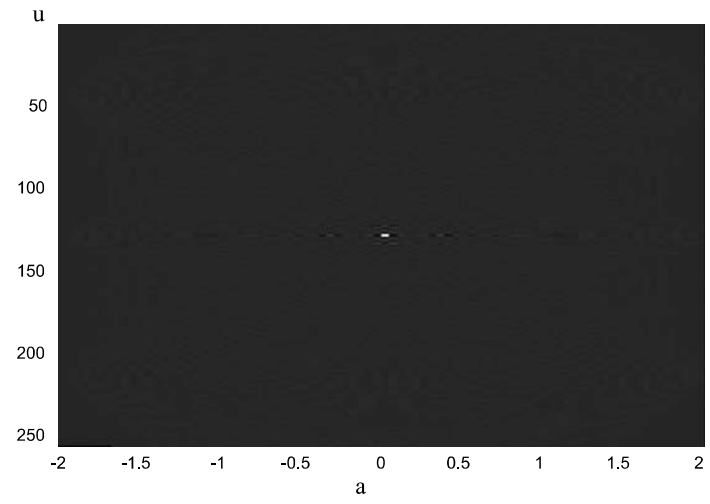

(b)

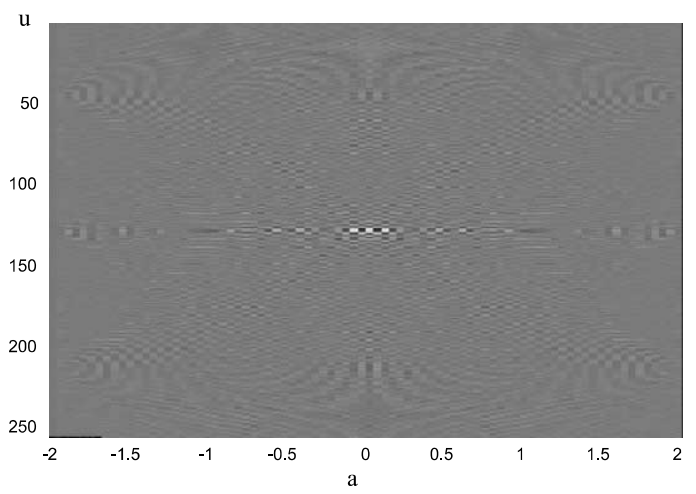

(e)

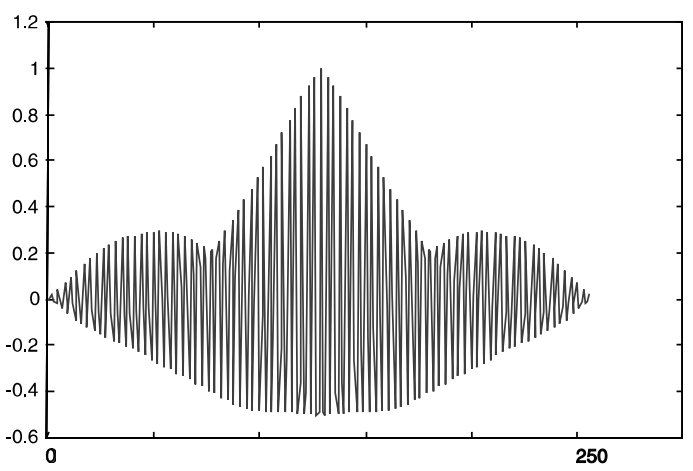

(c)

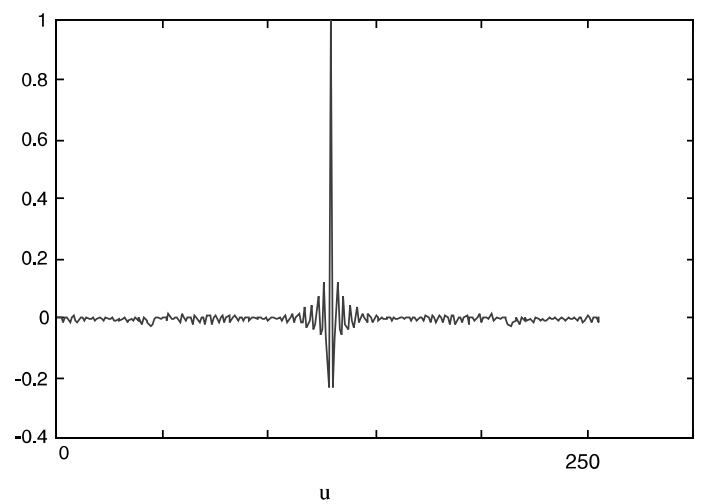

(d)

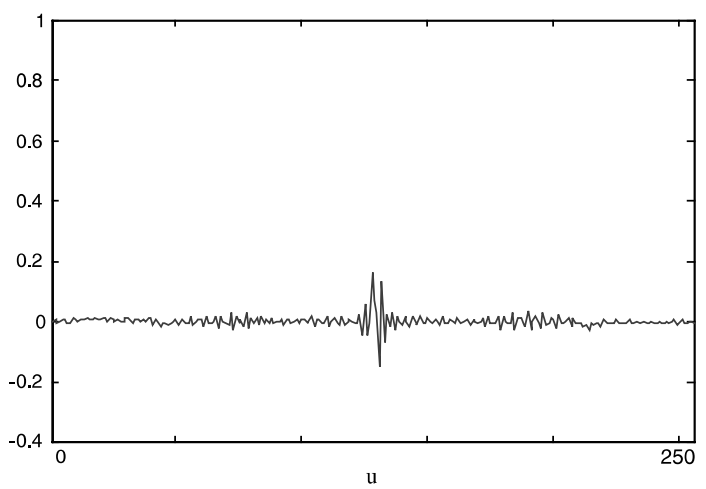

(f)

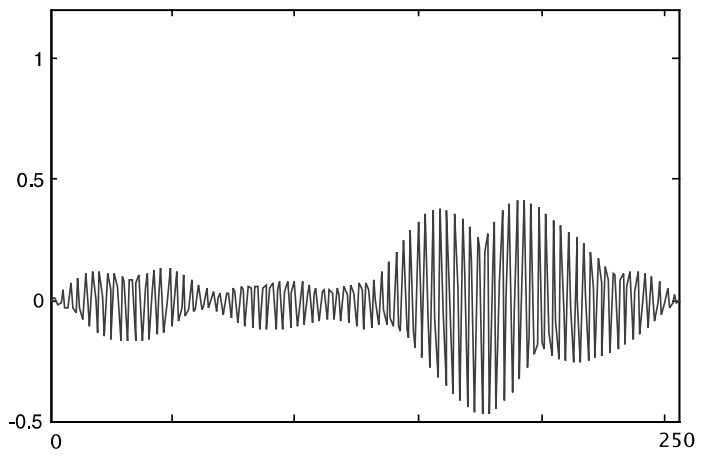

Fig. 2. (a) Correlation given by Eq. (4) when the input signal is equal to the reference signal, (b) correlation given by Eq. (4) when the input signal is equal to the second, different signal, (c) 1-D cross section of part a at $a=0$, (d) 1-D cross section of part b at $a=0$, (e) ordinary time-domain correlation of the reference signal with itself and (f) ordinary time-domain correlation of the reference signal with the second signal. [15].

\section{Computation considerations and experimental setup}

The Cartesian phase space $((x, p)$ chart $)$ is based on the FRT operation and thus may be computed using the fast Fourier transform (fft) algorithm. For an input signal having $N$ pixels, the computation complexity for computing the phase space is $M N \log N$ where $M$ is the computation resolution in the fractional order axis. The computation 
(a)

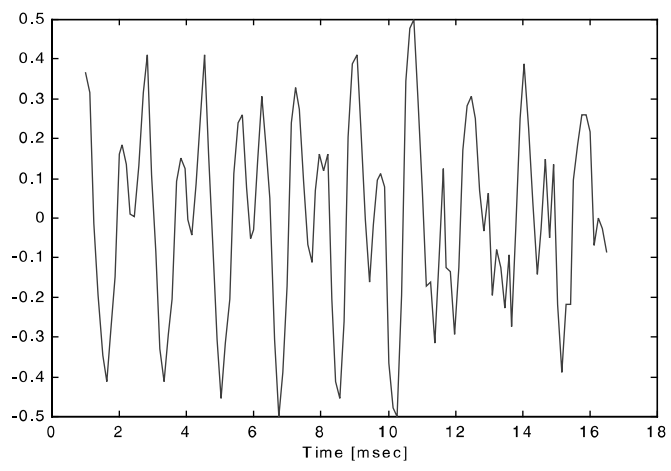

(b)

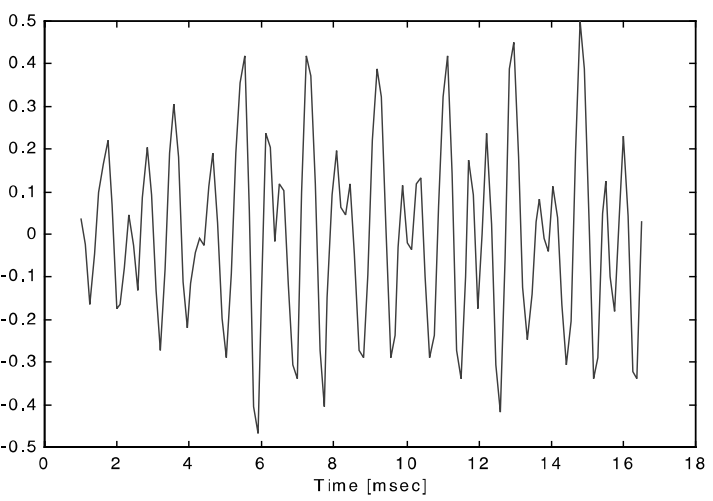

(c)

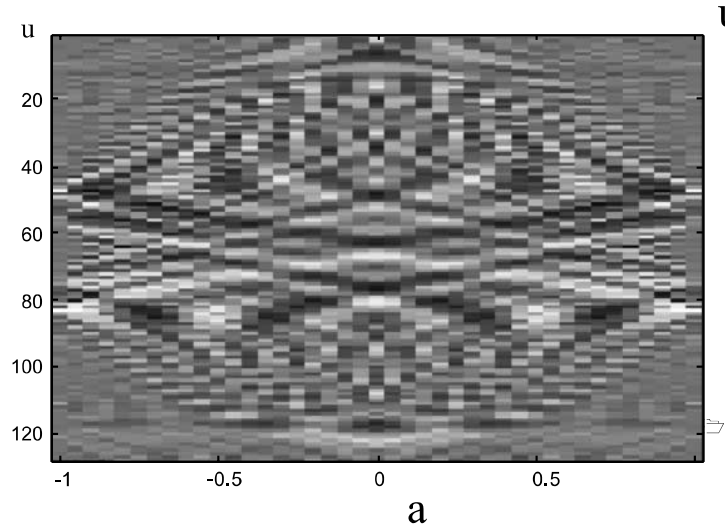

(d)

\section{u}

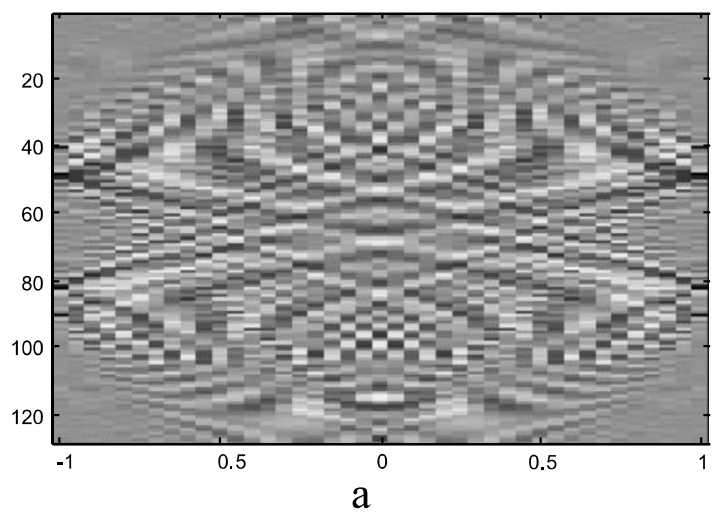

(e)

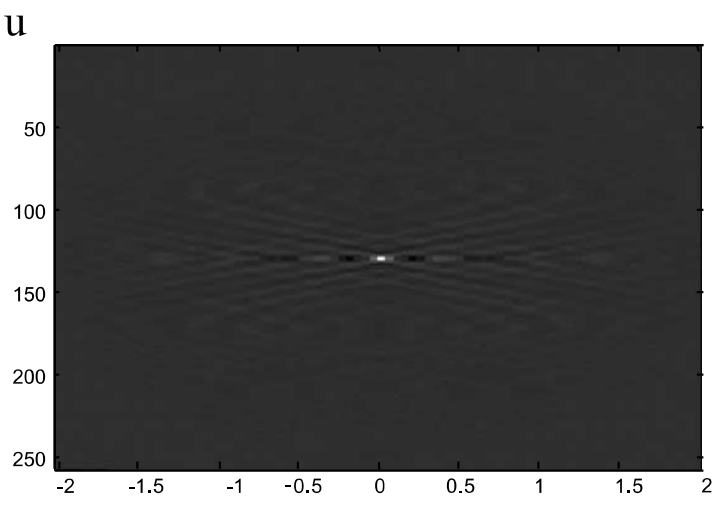

a

(f)

$\mathrm{u}$

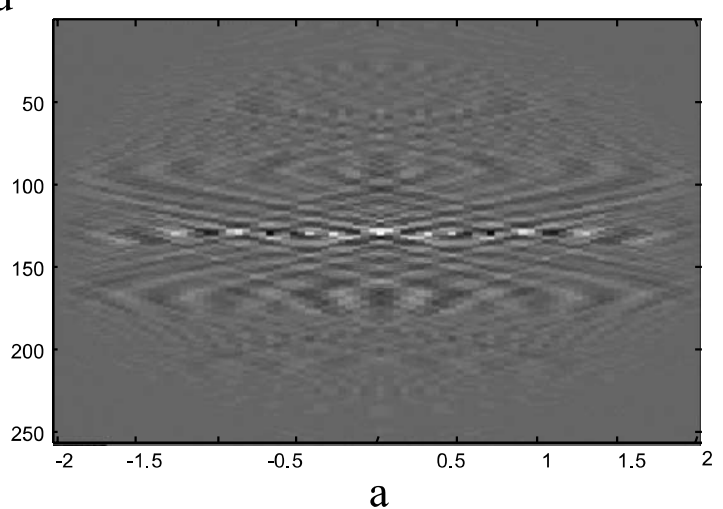

Fig. 3. (a) An input reference signal, (b) a different signal, (c) the Cartesian representation of the reference signal, (d) the Cartesian representation of the second signal, (e) auto correlation given by Eq. (4), (f) cross correlation given by Eq. (4), (g) 1-D cross section of part e at $a=0$, (h) 1-D cross section of part $\mathrm{f}$ at $a=0$, (i) ordinary time-domain auto correlation and (j) ordinary time-domain correlation of the reference signal with the second signal. 
(g)

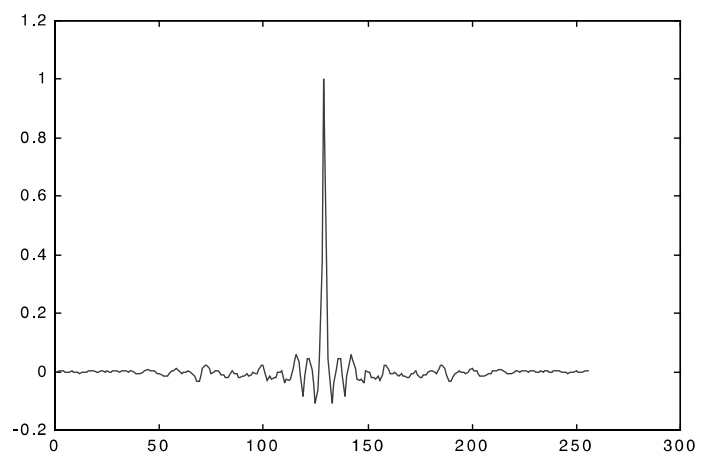

$\mathrm{u}$

(h)

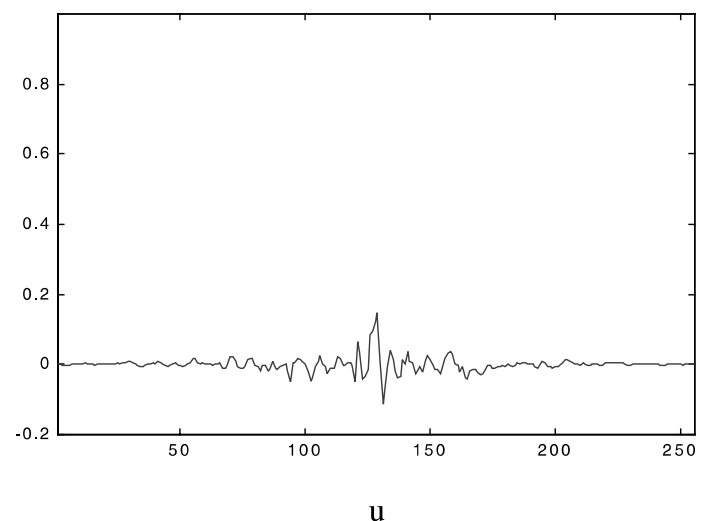

(i)

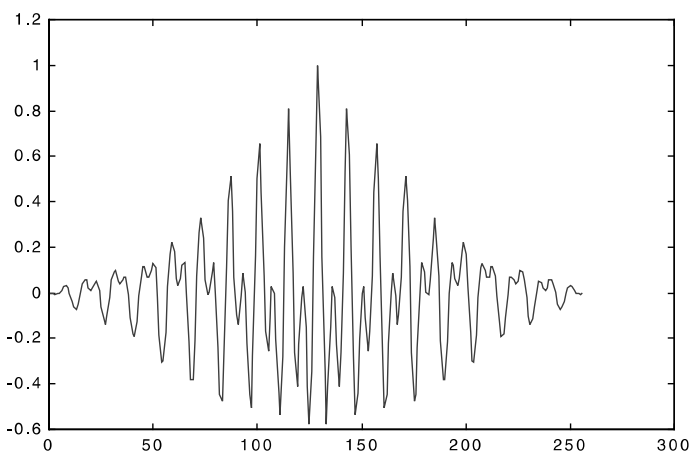

Fig. 3 (continued)

complexity for obtaining the 2-D correlation will be $M N \log (M N)$ since the correlation may also be obtained using the fft technique.

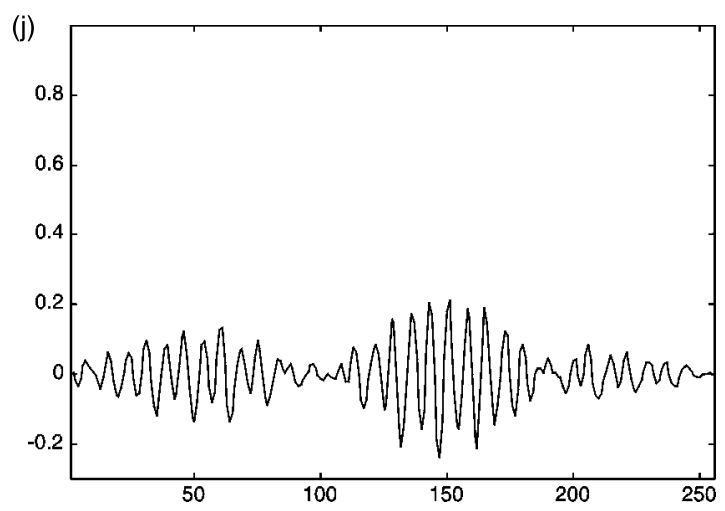

Fig. 3 (continued)

The phase space as well as the 2-D correlation may be obtained optically. As explained in Ref. [10], three filters are used in a configuration that implements an FRT with constant separation distances between the filters. Each filter contains strips that correspond to various fractional orders indicated as p1 to p25 in Fig. 4. Experimental results obtained from the above discussed setup are presented in Ref. [10]. An implementation of the 2-D correlation between the two-phase spaces may be obtained using a conventional VanderLugt 4-f correlator setup.

\section{Conclusions}

This paper presents a novel identification technique of fractional Fourier transform based phasespace representation. The proposed approach for detecting and identifying signals offers significant improvement with respect to common correlation techniques. The improvement is expressed both in obtaining a sharp and narrow auto correlation peaks with negligible side lobes and in a very low cross correlation peak of below $20 \%$ for all the cases. The conventional technique exhibits at least 10 times wider correlation peak with side lobes of more than $30 \%$ from peak's height and a cross correlation peak of above $50 \%$.

Exploring further possibilities in this direction, as well as the comparison of the proposed ap- 


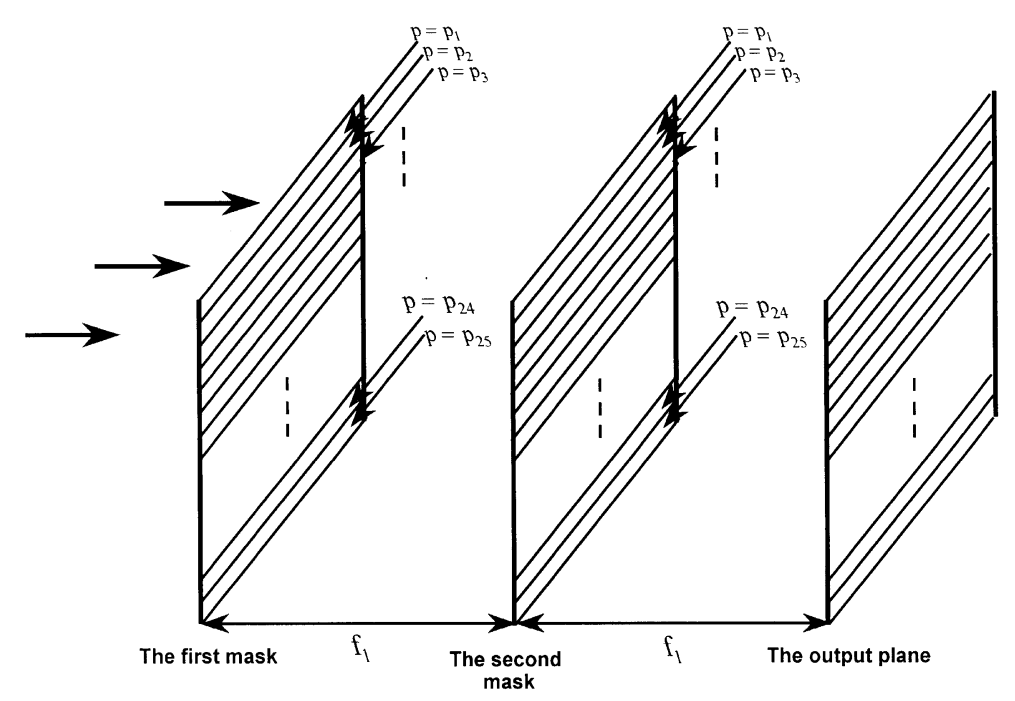

Fig. 4. Optical setup for realizing the $(x, p)$ chart.

proach with wavelet-based techniques constitute avenues for future research.

\section{References}

[1] H.M. Ozaktas, M.A. Kutay, D. Mendlovic, Introduction to the fractional Fourier transform and its applications, Advances in Imaging and Electron Physics, vol. 106, Academic Press, San Diego, 1999, pp. 239291.

[2] D. Mendlovic, H.M. Ozaktas, Fractional Fourier transformations and their optical implementation: I, J. Opt. Soc. Am. A 10 (1993) 1875-1881.

[3] H.M. Ozaktas, D. Mendlovic, Fractional Fourier transformations and their optical implementation: II, J. Opt. Soc. Am. A 10 (1993) 2522-2531.

[4] R.G. Dorsch, A.W. Lohmann, Y. Bitran, D. Mendlovic, H.M. Ozaktas, Chirp filtering in the fractional Fourier domain, Appl. Opt. 33 (1994) 7599-7602.

[5] H.M. Ozaktas, B. Barshan, D. Mendlovic, L. Onural, Convolution, filtering, and multiplexing in fractional Fourier domain and their relation to chirp and wavelet transforms, J. Opt. Soc. Am. A 11 (1994) 547-559.

[6] A.W. Lohmann, Image rotation, Wigner rotation and the fractional Fourier transform, J. Opt. Soc. Am. A 10 (1993) 2181-2186.

[7] H.M. Ozaktas, D. Mendlovic, Fractional Fourier optics, J. Opt. Soc. Am. A 12 (1995) 743-751.
[8] H.M. Ozaktas, M. Fatih Erden, Relationships among ray optical, Gaussian beam, and fractional Fourier transform descriptions of first-order optical systems, Opt. Commun. 143 (1997) 75-86.

[9] D. Mendlovic, Z. Zalevsky, R. Dorsch, Y. Bitran, A. Lohmann, H.M. Ozaktas, A new signal representation based on the fractional Fourier transform: definitions, J. Opt. Soc. Am. A 12 (1995) 4964-4971.

[10] D. Mendlovic, R.G. Dorsch, A.W. Lohmann, Z. Zalevsky, C. Ferreira, Optical illustration of a varied fractional Fourier transform order and the Radon-Wigner Display, Appl. Opt. 35 (1996) 3925-3929.

[11] H.M. Ozaktas, M.A. Kutay. Time-order signal representations. Proc. of the first IEEE Balkan Conf. on Signal Processing, Communications, Circuits and Systems, Bilkent Univ., Ankara., 2000.

[12] D. Mendlovic, Z. Zalevsky, H.M. Ozaktas, Applications of the fractional Fourier transform to optical pattern recognition, Optical Pattern Recognition, Cambridge University Press, 1998, pp. 89-125.

[13] R.K. Martinet, J. Morlet, A. Grossmann, Analysis of sound patterns through wavelet transforms, Int. J. Patt. Rec., Artificial Intell. 1 (2) (1987) 273-302.

[14] D. Mendlovic, Z. Zalevsky, I. Kiryuschev, G. Lebreton, Composite harmonic filters for scale, projection and shift invariant pattern recognition, Appl. Opt. 34 (1995) 310316.

[15] Adapted with permission from "The Fractional Fourier Transform with Applications in Optics and Signal Processing" H.M. Ozaktas, Z. Zalevsky, M.A. Kutay (John Wiley \& Sons Ltd. 2000). 